\title{
MEASURING IN WEIGHTED ENVIRONMENTS (Moving from Metric to Order Topology)
}

\author{
Claudio Garuti \\ Fulcrum Ingenieria Ltda. \\ claudiogaruti@fulcrum.cl
}

\begin{abstract}
This article addresses the problem of measuring closeness in weighted environments (decision-making environments). The relevance of this article is related with having a dependable cardinal measure of distance in weighted environments. Weighted environments is a non isotropic structure where the different directions (axes) may have different importance (weight) thus, there exist privilege directions. In this kind of structure would be very important to have a cardinal reliable index able to say how close or compatible is the set of measures of one individual with respect to the group or to anyone other. Common examples of this structure is the interaction between actors in a decision making process (system values interaction), matching profiles, pattern recognition, and any situation where a process of measurement with qualitative variables is involved.
\end{abstract}

Keywords: Weighted environments, Measurement, Compatibility, Order Topology.

\section{Introduction}

This article addresses the problem of measuring closeness in weighted environments (decisionmaking environments), using the concept of compatibility of priority vectors and value systems. For our purposes, compatibility is defined as the proximity or closeness between vectors within a weighted space. We will show a proposition for a compatibility index able to measure closeness in a weighted environment, thus, able to assess: pattern recognition; medical diagnosis support measuring the degree of closeness between disease-diagnosis profiles, Buyer-Seller matching profiles; measuring the degree of closeness between house buyer and seller projects or employment degree of matching; measuring the degree of closeness between a person's profile with the desired position profile; in curricula network design, Conflict Resolution; measuring closeness of two different value systems (ways of thinking) by identify and measuring the discrepancies, and in general measuring the degree of compatibility between any priority vectors in cardinal measure bases (order topology).

\section{2.- Literature Review}

In metric topology, the particular function of distance $\mathrm{D}(\mathrm{a}, \mathrm{b})$ is used to assess the closeness of two points $\mathrm{a}, \mathrm{b}$ as a real positive function that keeps 3 basic properties:

1.- $\mathrm{D}(\mathrm{a}, \mathrm{b})>0$ and $\mathrm{D}(\mathrm{a}, \mathrm{b})=0$ iif $\mathrm{a}=\mathrm{b}$ (definition of zero distance)

2.- $\mathrm{D}(\mathrm{a}, \mathrm{b})=\mathrm{D}(\mathrm{b}, \mathrm{a}) \quad$ (symmetry)

3.- $\mathrm{D}(\mathrm{a}, \mathrm{b})+\mathrm{D}(\mathrm{b}, \mathrm{c}) \geq \mathrm{D}(\mathrm{a}, \mathrm{c})$ (triangular inequality)

The general function of distance used to calculate the separation between two points is:

$D(a, b)=\operatorname{Lim}_{n \rightarrow k}\left(\sum_{i}\left(a_{i}-b_{i}\right)^{n}\right)^{1 / n} \quad(i=1, \ldots, n ; n=$ dimension of the space).

When applying different values of $\mathrm{k}$, different Norms of distance appear:

For $\mathrm{k}=1$, then: $\mathrm{D}(\mathrm{a}, \mathrm{b})=\sum_{\mathrm{i}} \mathrm{Abs}\left(\mathrm{a}_{\mathrm{i}}-\mathrm{b}_{\mathrm{i}}\right)$. Norm1, absolute Norm or path Norm; this Norm measure the distance from $a$ to $b$ within a 1D line, "walking" over the path, in one line-dimension.

For $\mathrm{k}=2$, then: $\mathrm{D}(\mathrm{a}, \mathrm{b})=\left[\left(\sum_{\mathrm{i}}\left(\mathrm{a}_{\mathrm{i}}-\mathrm{b}_{\mathrm{i}}\right)^{2}\right)\right]^{1 / 2}$. Norm2 or Euclidean Norm, this Norm measure the distance from $a$ to $b$, within a $2 \mathrm{D}$ plane (X-Y plane) getting the shortest path (the straight line).

For $\mathrm{k}=+\infty$, then: $\mathrm{D}(\mathrm{a}, \mathrm{b})=\operatorname{Max}_{\mathrm{i}}\left(\mathrm{abs}\left(\mathrm{a}_{\mathrm{i}}-\mathrm{b}_{\mathrm{i}}\right)\right)$. Norm $\infty$ or Norm Max; this Norm measure the distance from $a$ to $b$ within a $\infty \mathrm{D}$ hyperplane, getting the shortest path (the maximum coordinate) from all the possible paths.

\section{3.- Hypotheses/Objectives}

In order topology, measurement deals with dominance between preferences (intensity of preference), for instance: $\mathrm{D}(a, b)=3$, means that dominance or intensity of preference of " $a$ " over " $b$ " 
is equal to 3 , or that, $a$ is 3 times more preferred than $b$. When talking about preferences a relative absolute ratio scale is applied. Relative; because priority is a number created as a proportion of a total (percent or relative to the total) and has no needs for an origin or predefined zero in the scale. Absolute; because it has no dimension since it is a relationship between two numbers of the same scale leaving the final number with no unit. Ratio; because it is built in a proportional type of scale $(6 \mathrm{~kg} / 3 \mathrm{~kg}=2)$.

So, making a general analogy between the two topologies, one might say that: "Metric Topology is to Distance as Order Topology is to Intensity".

An equivalent concept of distance is presented in order to make a parallel between the three properties of distance of metric topology. This is applied in the order topology domain, considering a compatibility function (Eq.1) similar to distance function, but over vectors instead of real numbers.

Consideration: $\mathrm{A}, \mathrm{B}, \mathrm{C}$ are priority vectors of positive coordinates and $\Sigma_{\mathrm{i}} \mathrm{a}_{\mathrm{i}}=\Sigma_{\mathrm{i}} \mathrm{b}_{\mathrm{i}}=\Sigma_{\mathrm{i}} \mathrm{C}_{\mathrm{i}}=1$, and $\mathrm{G}(\mathrm{A}, \mathrm{B})$ is the compatibility function expressed as:

$$
\boldsymbol{G}(\mathrm{A}, \mathrm{B})=1 / 2 \Sigma_{\mathrm{i}}\left(\mathrm{a}_{\mathrm{i}}+\mathrm{b}_{\mathrm{i}}\right) \operatorname{Min}_{\mathrm{i}}\left(\mathrm{a}_{\mathrm{i}}, \mathrm{b}_{\mathrm{i}}\right) / \operatorname{Max}_{\mathrm{i}}\left(\mathrm{a}_{\mathrm{i}}, \mathrm{b}_{\mathrm{i}}\right)
$$

1.- $\quad 0 \leq G(\mathrm{~A}, \mathrm{~B}) \leq 1$ (Non negative real number)

The compatibility function $G$, returns a non negative real number that lays in the $0-1$ range. With $G(\mathrm{~A}, \mathrm{~B})=0$, if and only if $\mathrm{A}$ and $\mathrm{B}$ are perpendicular vectors $(\mathrm{A} \perp \mathrm{B})$, and represent the definition of total incompatibility between priority vectors $\mathrm{A}, \mathrm{B}$. $(\mathrm{A} \cdot \mathrm{B}=0)$, and $G(\mathrm{~A}, \mathrm{~B})=1$, if and only if $\mathrm{A}$ and $\mathrm{B}$ are parallel vectors, $(\mathrm{A}=\mathrm{B}$ for normalized vectors $)$, and represent the definition of total compatibility between priority vectors $A$ and $B$. $(A \cdot B=1)$

2.- $\quad G(\mathrm{~A}, \mathrm{~B})=G(\mathrm{~B}, \mathrm{~A})$ (Symmetry)

Symmetry condition, the compatibility measured from A to B is equal to the compatibility measured from B to A.

Easy to proof, just interchanging A for B and B for A in the compatibility function $G$.

3.- $\quad G(\mathrm{~A}, \mathrm{~B})+G(\mathrm{~B}, \mathrm{C}) \geq G(\mathrm{~A}, \mathrm{C})$ (Triangular inequality)

It is easy to prove that if $\mathrm{A}, \mathrm{B}$ and $\mathrm{C}$ are compatible priority vectors (i.e. $0.9 \leq G_{\mathrm{i}} \leq 1$ ), then property 3 is always satisfied. But, this property is also satisfied for the more relaxed (and interesting) condition where only two of the three vectors are compatible. For example, if $\mathrm{A}$ is compatible with $\mathrm{B}$ (i.e. $G(\mathrm{~A}, \mathrm{~B}) \geq 0.9)$ and if A is compatible with C (i.e. $G(\mathrm{~B}, \mathrm{C}) \geq 0.9$, or some other combination of $\mathrm{A}, \mathrm{B}$ and $\mathrm{C})$, then condition 3 is also satisfied. This more relaxed condition allows compatible and noncompatible vectors to be combined while property 3 is still satisfied.

This situation can be geometrically viewed in the next figure:

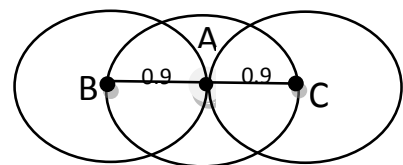

Figure A: Maximum circle of compatibility for position A, related to B and C

Figure A is showing the compatibility neighborhood for A, in relation with B and C, with its minimum compatibility value of 0.9 represented by the radius of the circle. (In the center the compatibility reaches its maximum value of 1.0 ). Thus, $G(\mathrm{~A}, \mathrm{~B})=G(\mathrm{~A}, \mathrm{C})=0.9$ is representing the minimum compatibility point, or the maximum distance for positions $\mathrm{B}$ and $\mathrm{C}$ to still be compatible with position A, of course, $G(\mathrm{~B}, \mathrm{C})<0.9$ they represents a non-compatible position for points $\mathrm{B}$ and $\mathrm{C}$. Notice that property $3 G(\mathrm{~A}, \mathrm{~B})+G(\mathrm{~B}, \mathrm{C}) \geq G(\mathrm{~A}, \mathrm{C})$ is still valid indeed, any combination that one can be made will keep the inequality satisfied, since if $C$ gets closer to $A$ (increasing the right side of the equation), then $G(\mathrm{~B}, \mathrm{C})$ will also grow. The extreme case when $\mathrm{C}$ is over $\mathrm{A},(G(\mathrm{~A}, \mathrm{C})=1.0)$ then $G(\mathrm{~B}, \mathrm{~A})+G(\mathrm{~B}, \mathrm{C})=0.9+0.9=1.8>1.0$ keeping the inequality satisfied.

We may also define incompatibility function as the arithmetic complement of the compatibility: Incompatibility $=1-$ Compatibility. Thus: Incompatibility is equivalent to $1-G$. By 
the way, the incompatibility concept is more close to the idea of distance, since the greater the distance the greater the incompatibility.

Two simple examples of this parallel between $\mathrm{D}(\mathrm{x}, \mathrm{y})$ and $G(\mathrm{X}, \mathrm{Y})$ are given. But first, to make D and G functions comparable, D must be transformed into relative terms as a percent value since the priority vectors are normalized vectors for $\mathrm{G}$ function. Thus, the maximum possible value for $\mathrm{D}^{1}$ (Norm1) is 2 and for $\mathrm{D}^{2}$ (Norm2) is $\sqrt{2}$, while performing the ratios with respect to the maximum possible value and obtaining $\mathrm{D}$ in relative terms as percent of the maximum value.

For the first example, two different and very different vectors A and B with coordinate: $\{0.3$, $0.7\}$ with $\{0.7,0.3\}$ and $\{0.1,0.9\}$ with $\{0.9,0.1\}$ are considered.

Considering also Incompatibility= $1-$ Compatibility: $1-G(\mathrm{~A}, \mathrm{~B})$. Then:

Compatibility between $\mathrm{A}$ and $\mathrm{B}$ is shown by $G(\mathrm{~A}, \mathrm{~B})$ (Real positive value laying in 0-1 range.) Incompatibility between $\mathrm{A}$ and $\mathrm{B}$ is shown by $1-G(\mathrm{~A}, \mathrm{~B})$ (Real positive value laying in 0-1 range also). Table $1 \mathrm{a}$ shows the results of applying $\mathrm{D}^{1}, \mathrm{D}^{2}$ and $(1-G)$ functions.

\begin{tabular}{|c|c|c|c|}
\hline $\begin{array}{c}\text { A,B } \\
\text { Coordinates }\end{array}$ & $\begin{array}{c}\mathrm{D}^{1}(\mathrm{a} . \mathrm{b}) \\
\text { Distance A-B in } \\
\text { Norm1 (normalized) }\end{array}$ & $\begin{array}{c}\mathrm{D}^{2}(\mathrm{a}, \mathrm{b}) \\
\text { Distance A-B in } \\
\text { Norm2 (normalized) }\end{array}$ & $\begin{array}{c}\text { Incompatibility= } \\
1-G(\mathrm{~A}, \mathrm{~B})\end{array}$ \\
\hline \begin{tabular}{c|c|c|}
$\mathrm{A}=\{0.3,0.7\}$ \\
$\mathrm{B}=\{0.7,0.3\}$
\end{tabular} & $0.8 / 2=0.4(40 \%)$ & $0.4 \sqrt{ } 2 / \sqrt{ } 2=40 \%$ & $1-(0.3 / 0.7)=57 \%$ \\
\hline \begin{tabular}{c|c|c}
$\mathrm{A}=\{0.1,0.9\}$ \\
$\mathrm{B}=\{0.9,0.1\}$
\end{tabular} & $1.6 / 2=0.8(80 \%)$ & $0.8 \sqrt{2} / \sqrt{ } 2=80 \%$ & $1-(0.1 / 0.9)=89 \%$ \\
\hline
\end{tabular}

Table 1a: Evaluating distance and incompatibility for non-similar set of coordinates

Figure B shows in 2D Cartesian axes how distant (incompatible) is A from B in both cases. Notice that for the case represented in brown (for $\mathrm{D}=80 \%$ and $\mathrm{G}=89 \%$ ), vectors $\mathrm{A}$ and $\mathrm{B}$ are (geometrically) almost in a perpendicular position (A relative to $\mathrm{B}$ ).

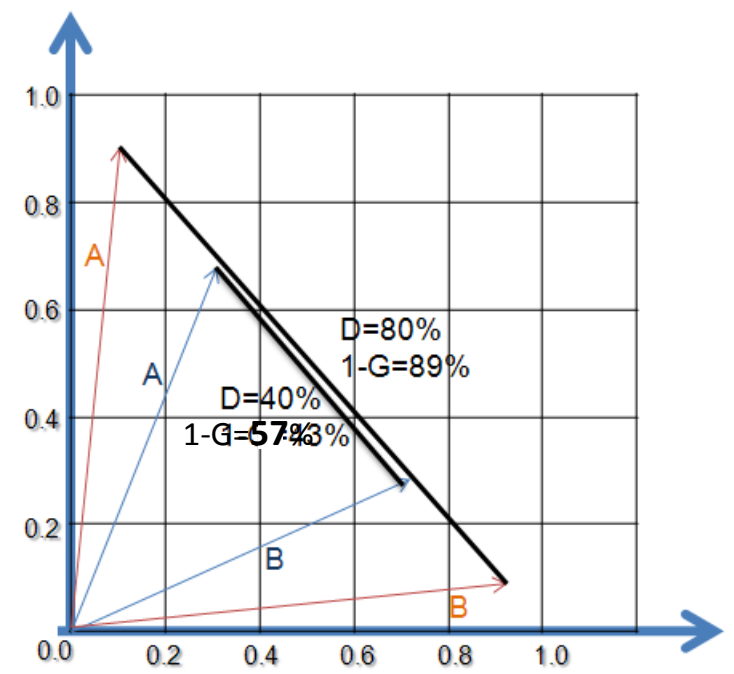

Figure B: Two examples for distance and compatibility functions for far and very far A and B

Next, using the same process, we compare for similar and very similar A, B vectors with coordinates: $\{0.3,0.7\}$ compared with $\{0.4,0.6\}$ and $\{0.10,0.90\}$ compared with $\{0.11,0.89\}$.Table $1 \mathrm{~b}$ shows the results of applying $\mathrm{D}^{1}, \mathrm{D}^{2}$ and $1-G$ (Incompatibility $=1$ - Compatibility).

\begin{tabular}{|c|c|c|c|}
\hline A,B Coordinates & $\mathrm{D}^{1}(\%)$ & $\mathrm{D}^{2}(\%)$ & Incompatibility= 1-G(\%) \\
\hline $\mathrm{A}=\{0.30,0.70\}$ & $0.2 / 2=0.1(10 \%)$ & $0.1 \sqrt{2} / \sqrt{2}=10 \%$ & $1-0.820=18 \%$ \\
\hline
\end{tabular}




\begin{tabular}{|l|l|l|l|}
\hline $\mathrm{B}=\{0.40,0.60\}$ & & \\
\hline $\begin{array}{l}\mathrm{A}=\{0.10,0.90\} \\
\mathrm{B}=\{0.11,0.89\}\end{array}$ & $0.02 / 2=0.01(1 \%)$ & $0.01 \sqrt{ } 2 / \sqrt{ } 2=1 \%$ & $1-0.981=1.9 \%$ \\
\hline
\end{tabular}

Table 1b: Calculating distance and incompatibility for similar set of coordinates

The trend of the results for $\mathrm{D}$ and $G$ functions is the same in both cases, when increasing the distance or making vectors more perpendicular and when decreasing the distance or making the vectors more parallel. This is an interesting parallel to these concepts and their trends, considering that different concepts (distance and incompatibility in different ratio scales) are being used.

\section{4.- Research Design/Methodology}

One way to calculate compatibility in a general form is by using the inner or scalar vector product, defined as: $A \cdot B=|A||B| C o s \alpha$. This expression of dot product is preferable to the Cartesian version, since it highlights the relevance of the projection concept represented by Cos $\alpha$ and also because when working with normalized vectors the expression $A \cdot B$ become equal to $\operatorname{Cos} \alpha$, which shows that the projection part of the dot product is the relevant part.

Definitions and Conditions

Assuming:

A- Two normalized vectors are close (compatible), when the angle ( $\alpha$ ) formed by both vectors on the hiperplane is near $0^{\circ}$ or $\operatorname{Cos} \alpha$ is near to one. From a geometric point of view, they will be represented by parallel or nearly parallel vectors. In this case they will be defined as compatible vectors.

B- Two normalized vectors are not close (not compatible) when the angle $(\alpha)$ formed by both vectors on the hiperplane is near $90^{\circ}$ or Cos $\alpha$ is near to zero. From a geometric point of view, they will be represented by perpendicular or nearly perpendicular vectors. In this case they will be defined as non-compatible vectors.

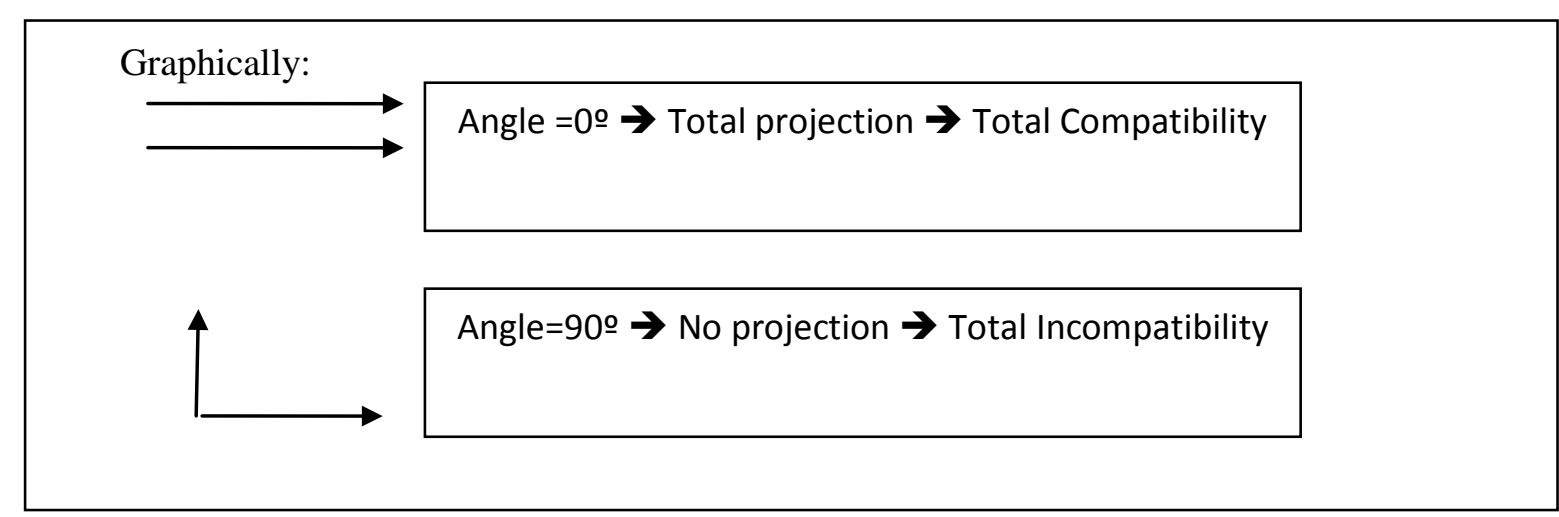

Figure E: Geometric interpretation of vector compatibility in terms of its projection

Figure E shows the geometric interpretation of vector compatibility. Therefore, there is an operative way to measure compatibility in terms of vector projection. This interpretation of dot product will be very useful for the purposes of compatibility measurement of two priority vectors in the domain of order topology.

Since the space is weighted, it is also necessary to weight each projection (each $\operatorname{Cos} \alpha_{i}$ ) and to take into account the changes of the angle (projection) and weight coordinate by coordinate (coordinate i may have a different projection and weight of coordinate $\mathrm{i}+1$ ). Thus, the final formula to assess a general compatibility index of two consistent vectors A, B from point to point throughout the both profiles is:

$$
\begin{aligned}
& \boldsymbol{G}(\mathrm{A}, \mathrm{B})=1 / 2 \Sigma_{\mathrm{i}}\left(\mathrm{a}_{\mathrm{i}}+\mathrm{b}_{\mathrm{i}}\right) \operatorname{Min}_{\mathrm{i}}\left(\mathrm{a}_{\mathrm{i}}, \mathrm{b}_{\mathrm{i}}\right) / \operatorname{Max}_{\mathrm{i}}\left(\mathrm{a}_{\mathrm{i}}, \mathrm{b}_{\mathrm{i}}\right) \\
& \text { With: } \quad \sum \mathrm{a}_{\mathrm{i}}=\Sigma \mathrm{b}_{\mathrm{i}}=1
\end{aligned}
$$


This can be shown graphically in Figure F.

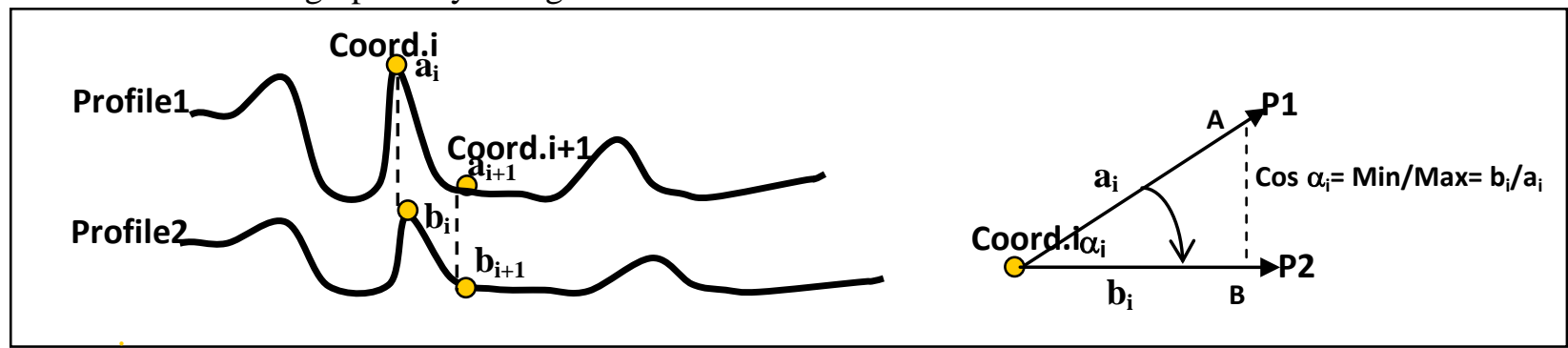

Figure F: Representation of the cosine projection changing point to point in terms of the profiles

The $\boldsymbol{G}$ function is a transformation function that takes positive real numbers from the range $[0,1]$, coming from normalized vectors $\mathrm{A}, \mathrm{B}$ and returns a positive real number on the same range: $\mathrm{a}_{\mathrm{i}}, \mathrm{b}_{\mathrm{i}} \mathbf{X}[0,1] \rightarrow \boldsymbol{G}(\mathrm{A}, \mathrm{B}) \rightarrow R^{+} \mathbf{X}[0,1]$

This transformation has two particularly good properties:

1. It is bounded (presenting no singularities or divergence).

2. The outcome is very easily interpreted as a percentage of compatibility, representing the $0=0 \%$, or total incompatibility and $1=100 \%$, or total compatibility.

It is also possible to define a threshold for compatibility index at $90 \%$. Thus, when two vectors have an index of compatibility equal or greater than $90 \%$, they should be considered compatible vectors. Since, Incompatibility= 1 - Compatibility, then the threshold for tolerable incompatibility is $10 \%$.

Next, a simple application example for 2D vectors A, B is presented.

The first case is for $\mathrm{A}=\mathrm{B}$.

$\mathrm{A}=\{0.5 ; 0.5\} ; \mathrm{B}=\{0.5 ; 0.5\}$, then:

$G(\mathrm{~A}, \mathrm{~B})=1 / 2((0.5+0.5)(1 / 1)+(0.5+0.5)(1 / 1))=1$

Being $100 \%$ compatible or $1-1=0 \%$ incompatible, this result is expected because A and B is the same vector.

The second case is for two very different vectors.

$\mathrm{A}=\{0.10 ; 0.90\} ; \mathrm{B}=\{0.90 ; 0.10\}$, then:

$\operatorname{Min}\{a 1 ; b 1\}=\operatorname{Min}\{0.1 ; 0.9\}=0.1 ; \operatorname{Max}\{a 1 ; b 1\}=\operatorname{Max}\{0.1 ; 0.9\}=0.9$, and $(\operatorname{Min} / \operatorname{Max})_{1}=0.111$

The same for coordinate 2 :

$(\operatorname{Min} / \operatorname{Max})_{2}=0.111, \quad$ and $\quad G(\mathrm{~A}, \mathrm{~B})=1 / 2((0.1+0.9) 0.111+(0.9+0.1) 0.111)=0.111 . \quad(11.1 \% \quad$ of compatibility or $88.9 \%>>10 \%$ of incompatibility). This is also an expected outcome since geometrically they are almost perpendicular vectors (vector A has almost no projection over B).

\section{5.- Limitations}

It should be noted that when using other compatibility index formulas, for instance, the classic dot product and dividing the result by $n$ (like taking the average instead of weighting to evaluate the incompatibility), the result of the operation is: $(0.1 / 0.9+0.9 / 0.1) / 2=4.55$ (or $355 \%$ of incompatibility or deviation from n). Doing the same in a matrix environment, that is forming the Hadamard product and dividing by $n^{2}$ instead of $n$ to assess the deviation, the result is $83,012 / 2^{2}=$ $20.75\left(20.75-1=19.75\right.$ or $1975 \%$ of incompatibility or deviation from $n^{2}$. Presenting both situation a singularity problem (not bounded function).

Another formula to evaluate incompatibility is the Hilbert formula, which expression is: $\log \left(\operatorname{Max}_{\mathrm{i}}(\mathrm{a}, \mathrm{b}) / \operatorname{Min}_{\mathrm{i}}(\mathrm{a}, \mathrm{b})\right)=1.909$, $(191 \%$ of incompatibility). These last three formulae present a divergence process (singularities). As vector A deviates from vector $\mathrm{B}$, (being more and more incompatible), they become close to the perpendicular vectors. The $G$ function becomes the only formula capable of correctly assessing the compatibility without falling in a singularity (divergence), 
or remaining immobilized, as a standard distance calculation does when the absolute difference between the coordinates is kept constant. In fact, a complete study ${ }^{1}$ of the behaviour of different compatibility indices is presented in Table $3^{2}$. The study was made initially for a 2D space (vectors of two coordinates), since the idea was to perform a sensitive analysis and observe the patterns of behavior of different compatibility indices in two special situations:

A. Parallel trend situation: when two vectors are near each other or close enough to be parallel vectors.

B. Perpendicular trend situation: when two vectors are separate or close enough to be perpendicular vectors.

Table2 summarizes the compatibilities indices that exist, adding the option of Euclidian norm (the classic distance calculation based on Norm2), normalized by its maximum possible value of $\sqrt{2}$ to present the results in percentage format.

\begin{tabular}{lll|}
\hline Hilbert formula (Hilbert's index): & $C(A, B)=\log \left\{\operatorname{Max}_{i}\left(a_{i} / b_{i}\right) / \operatorname{Min}_{i}\left(a_{i} / b_{i}\right)\right\}$ \\
Inner vector product inverted (IVP): & $C(A, B)=\{A\}^{\circ}\{B\} / n=\left(\Sigma_{i} a_{i} \times 1 / b_{i}\right) / n$ \\
Weighted inner vector product (WIVP): & $C(A, B)=\{A\}\{B\}\{W\}=\Sigma_{i}\left(a_{i} \times 1 / b_{i} \times W_{i}\right)$ \\
Hadamard product (Saaty's índex): & $C(A, B)=[A]^{\circ}[B]^{t} / n^{2}=\left[\Sigma_{i} \Sigma_{j} a_{i j} \times 1 / b_{i j}\right] / n^{2}$ \\
GCI normalized (G index): & $C(A, B)=1 / 2 \Sigma_{i}\left(a_{i}+b_{i}\right)\left(\operatorname{Min}\left(a_{i j}, b_{i}\right) / \operatorname{Max}\left(a_{i}, b_{i}\right)\right.$ \\
Euclidian formula (normalized): & $C(A, B)=\operatorname{SQRT}\left(1 / 2 \Sigma_{i}\left(a_{i}-b_{i}\right)^{2}\right)$ \\
\hline
\end{tabular}

Table2: Definition of different formulas for compatibility assessment

Six formulae in a 2D vector for 7 cases for two different trends (parallel trend and perpendicular trend) were tested. The results are shown in Table 3.

\begin{tabular}{|c|c|c|c|c|c|c|c|}
\hline & CASE SENS & ILITATIOI & N TABLE (FOR & HOMOG & ENEUS VE & CTORS) & \\
\hline CASE & Vector & ord. & Hadamard & $\mathbf{G}$ & Hilbert & IVP & Euclidean \\
\hline Paralell Trend & & & & Distance o & I Incompat & ibility & \\
\hline 0 & 0,5 & 0,5 & $1,010 \%$ & $9,523 \%$ & $\mathbf{8 , 7 1 5} \%$ & $1,010 \%$ & $4,999 \%$ \\
\hline & 0,45 & 0,55 & & & & & \\
\hline 1 & 0,533 & 0,467 & $0,058 \%$ & $2,371 \%$ & $2,097 \%$ & $0,218 \%$ & $1,202 \%$ \\
\hline & 0,545 & 0,455 & & & & & \\
\hline 2 & 0,633 & 0,367 & $0,068 \%$ & $2,370 \%$ & $2,259 \%$ & $0,760 \%$ & $1,202 \%$ \\
\hline & 0,645 & 0,355 & & & & & \\
\hline 3 & 0,733 & 0,267 & $0,097 \%$ & $2,363 \%$ & $2,702 \%$ & $1,548 \%$ & $1,202 \%$ \\
\hline & 0,745 & 0,255 & & & & & \\
\hline 4 & 0,833 & 0,167 & $0,198 \%$ & $2,348 \%$ & $3, \mathbf{8 6 0} \%$ & $3,161 \%$ & $1,202 \%$ \\
\hline & 0,845 & 0,155 & & & & & \\
\hline 5 & 0,933 & 0,067 & $1,108 \%$ & $2,285 \%$ & $9,126 \%$ & $10,274 \%$ & $1,202 \%$ \\
\hline & 0,945 & 0,055 & & & & & \\
\hline 6 & 0,987 & 0,013 & $280,851 \%$ & $1,839 \%$ & $111,919 \%$ & $599,399 \%$ & $1,202 \%$ \\
\hline & 0,999 & 0,001 & & & & & \\
\hline 7 & 0,999 & 0,00100 & $2452,727 \%$ & $0,149 \%$ & $288,412 \%$ & $4949,950 \%$ & $0,100 \%$ \\
\hline & 0,99999 & 1E-05 & & & & & \\
\hline Perpendicular & ar trend: & & & Distance o & Incompat & ibility & \\
\hline 0 & 0,5 & 0,5 & $0,278 \%$ & $7,250 \%$ & $4,576 \%$ & $5,556 \%$ & $3,535 \%$ \\
\hline & 0,5 & 0,45 & & & & & \\
\hline 1 & 0,533 & 0,467 & $2,762 \%$ & $14,832 \%$ & $14,369 \%$ & $0,644 \%$ & $8,337 \%$ \\
\hline & 0,455 & 0,555 & & & & & \\
\hline 2 & 0,633 & 0,367 & $36,322 \%$ & $43,504 \%$ & $49,607 \%$ & $17,605 \%$ & $27,803 \%$ \\
\hline & 0,355 & 0,645 & & & & & \\
\hline 3 & 0,733 & 0,267 & $153,633 \%$ & $67,600 \%$ & $9042,100 \%$ & $61,645 \%$ & $47,800^{\circ}$ \\
\hline & 0,255 & 0,745 & & & & & \\
\hline 4 & 0,833 & 0,167 & $630,739 \%$ & $80,808 \%$ & $143,445 \%$ & $178,561 \%$ & $67,797 \%$ \\
\hline & 0,155 & 0,845 & & & & & \\
\hline 5 & 0,933 & 0,067 & $5931,685 \%$ & $93,500 \%$ & $237,880 \%$ & $751,727 \%$ & $87,801 \%$ \\
\hline & 0,055 & 0,945 & & & & & \\
\hline 6 & 0,987 & 0,013 & $1896128,846 \%$ & $99,291 \%$ & $487,994 \%$ & $49250,651 \%$ & $\mathbf{9 8 , 5 9 0 \%}$ \\
\hline & 0,001 & 0,999 & & & & & \\
\hline 7 & 0,99999 & 0,00001 & $249994999976 \%$ & $\mathbf{9 9 , 9 9 9 \%}$ & $999,999 \%$ & & $99,999 \%$ \\
\hline & 0,00001 & 0,99999 & & & & & \\
\hline
\end{tabular}

Table 3: Sensitive analysis for different compatibility indices

\footnotetext{
${ }^{1}$ Claudio Garuti, “When close really means close?” Paper of Compatibility. Presented in the ISAHP9 Symposium and Proceeding. Viña del Mar-Chile, 2007.

${ }^{2}$ The different compatibility indexes analyzed were taken from the literature and from linear algebra definitions.
} 


\section{6.- Conclusions}

There are two global conclusions of this study:

First, that we need some kind of distance measurement (proximity or compatibility) in weighted environments (order topology domain), in order to mathematically define if two profiles are really close or not, this will make possible a matching analysis process, considering that those profiles may represent: system values, a pattern recognition process, benchmarking, or even a membership analysis (closeness analysis).

Second, this analysis shows that the only compatibility index that performs correctly for every case is the $G$ index, keeping the outcome always in the $0-100 \%$ range (like the normalized Euclidean formula), and this is an important condition, since any value out of the $0-100 \%$ range would be difficult to interpret (and the beginning of a possible divergence). It is also important to note the $G$ and Euclidean outcomes in Table 3.They are close , but $G$ is much more accurate or sensitive to changes since they are not based on absolute differences $\left(\Delta \mathrm{x}_{\mathrm{i}}\right)$ as distance is, but on relative absolute ratios scale (the Min/Max weighted projection). Indeed, the Euclidean distance calculation shows no differences in the distance of parallel trend from case 1 to 6 (Euclidean based index cannot detect the difference in the compatibility value) because the absolute difference of the coordinates remains the same. Therefore, with the Euclidian based index one may reach the wrong conclusion that no difference exists for vector compatibility from cases 1 to 6 (the first case study is as incompatible as $2,3,4,5$ or 6 , which is not an expected result). This unexpected behaviour occurs because the Euclidean norm is based on differences, and because it's not concerned about the weights of the coordinates and the projections between vectors. It is important to remember that the numbers inside the priority vector represent preferences, hence, in terms of proximity, it is better to be close to a big preference (big coordinate) than to the small ones. Other tests made in greater spaces (3D to 10D) show the same trend. Moreover, the bigger the space dimension, the greater the likelihood of finding singularity points for the others formulae in both trends parallel and perpendicular.

It is interesting to note that function $G$ depends on two different dimensional factors, on one side the intensity of preference (related with the weight), and on the other side the angle of projection between the vectors (profiles). This means that $G$ is a function of the intensity (I) of preference and the angle $(\alpha)$ between the priority vectors, that is: $(G=f(I, \alpha))$. Clearly, the $G$ function is not the simple dot product (as normally defined), but something more complex and rich. It is also important to note that both data (intensity and angle) are implicit in the coordinates of the priority vectors (the profiles) and have to be correctly extracted if one wants to produce a good and reliable index to measuring the degree of compatibility or closeness between profiles in weighted environments.

\section{7.- Key References}

1. Garuti, C. A. 2007. Measuring compatibility (closeness) in weighted environments: when close really means close? Int. Symposium on AHP, 9, Viña del Mar, 2007.

2. Garuti, C. A. 2012. Measuring in weighted environments, moving from metric to order topology. Santiago, Chile: Universidad Federico Santa Maria.

3. Saaty, T. L. 2006. Fundamentals of decision making and priority theory. 2 ed. Pittsburgh: RWS.

4. Whitaker, R. 2007. Validation examples of the Analytic Hierarchy Process and Analytic Network Process. Mathematical and Computer Modelling. 2007, Vol. 46, pp. 840-859. 